\title{
EDITORIAL
}

\section{Medical Training in the Army from First Aid to Advanced Trauma Life Support The Development of an Integrated Package}

All ranks in the Army receive some form of medical training, ranging from the basic first aid of ATD5 to the higher professional training necessary for accreditation for consultant appointments. In the past this training has been fragmented and compartmentalised and has led to the implied criticism that it is out of date (1).

In civilian practice the management of trauma, which is the business of military medical services, has been completely overhauled with the inception of Advanced Trauma Life Support (ATLS) and the adoption of strict protocols for the management of the severely injured. The military response has been the Battlefield Advanced Trauma Life Support (BATLS) course which was initially aimed at medical officers but is now 'being adapted for all medical personnel in the form of a Battlefield Advanced Resuscitation and Technical Skills (BARTS) course. A further variant, the Covert Advanced Trauma Life Support (CATLS) course is being developed for the Special Forces to meet their particular needs. An inaugural pilot course has been successfully run with warm approval from that body.

Research into civilian trauma has indicated that there is a "golden hour" in which most avoidable deaths occur, after which the mortality remains fairly constant until the familiar "six hour rule" is exceeded. Within this "golden hour" deaths arise from Airway, Breathing and Circulatory problems in that order and (neurological) Disability the $A B C D$ with which we are becoming familiar. It is the management of the casualty within this "golden hour" to which much effort has been addressed. ATLS training lays protocols for the hospital management of the victim of severe trauma. BATLS has used the ATLS model to develop similarly didactic protocols for the pre-hospital care of serious casualties arising on the battlefield. BARTS training will achieve the same protocols (dare one say drills) for the military paramedic.

These principles have been adopted by the civilian bodies which formulate policy for first aid training (Resuscitation Council [UK], Royal College of Surgeons [ATLS Committee], British Red Cross and St John Ambulance) and are being implemented by the voluntary first aid societies.

What then is the military response? Firstly the military position in dealing with severe trauma is different from that in civilian practice. In the heat of battle the primary aim is to seize the objective with the management of casualties being at best a secondary aim. Secondly the military doctor is likely to be faced, at an early stage of his career, with a number of serious casualties in the most inhospitable of surroundings where the civilian model of deploying a number of highly skilled specialists to a single casualty is not appropriate. Highly skilled medical support is a scarce and expensive resource, which should be concentrated in appropriately sited hospitals and should not be scattered around the battlefield in penny packets even if resources did permit.

Having recognised the differences in the military requirement, how do we tailor the resources to meet them?

Firstly, we need to define exactly what we want. On the battlefield we require advanced life support skills concentrating upon problems relating to the airway, breathing, circulation and neurological disability, and their management. In peacetime the requirement is to attempt to respond to the peacetime expectations of medical care, and so we teach a militarised version of the first aid taught by the voluntary societies. A glance into any first aid box, be it civilian or military will highlight our dilemma. Whilst there will be plenty of equipment capable of dealing with minor injuries most pay no attention to the problem of severe trauma, presumably in the expectation that skilled assistance will rapidly arrive. It is quite clear that we need both the "ATLS" type of skills even at the most basic level as well as the more conventional first aid skills we have to come to associate with the voluntary societies. Thus we have the ATD5 syllabus and training objectives which have been rewritten to incorporate ATLS terminology by changing the emphasis from the " $4 \mathrm{Bs}$ " (Breathing, Bleeding, Breaks and Burns) to the ABCD (Airway, Breathing, Circulation and [neurological] Disability) format. For the first time at this level, consideration of the possibility of a cervical spine injury is acknowledged and techniques for maintaining an airway with the neck in the neutral position are taught such as the chin lift and jaw thrust. It is a start but it still falls between the two extremes mentioned above.

Secondly, the First Aid Handbook, so long awaited as part of the Aid to Save package has been rewritten and retitled Battlefield First Aid. It now incorporates the new terminology and techniques and is intended, like Survive to Fight and Shoot to Kill, as an aide-memoire rather than a replacement for ATD5 training.

At this level, although they will never be prime lifesaving techniques on the battlefield, Exhaled Air Resuscitation and Chest Compression are retained purely because in this day and age it would be negligent not to.

Also at this level the question of the primary survey is raised. The thrust of ATD5 training is to attempt to teach a series of first aid drills, so rather than teach the primary survey per se, the soldier is taught to recognise and manage: the obstructed Airway, Breathing problems, Circulatory problems and basic neurological Disability and to 
Evacuate the casualty the ABCDE of ATLS. In effect this amounts to the Primary Survey and initial Resuscitation phases of ATLS, carried out forward whilst deferring the Secondary Survey and Definitive care phases until rearward evacuation to third line and beyond has been achieved. The resulting military application of the ATLS philosophy is unique but may indeed have relevance to those who seek to apply the principles of ATLS to disaster relief.

Turning to the "professional medic" - the medically trained soldier - the training objectives have been rewritten to incorporate the tenets of ATLS and to use the new terminology. The fully trained Combat Medical Technician is capable of performing advanced life-saving skills such as endotracheal intubation, cricothyrotomy, insertion chest drains and the setting up of IV infusions as well as conducting a primary survey and acting upon the findings as stated above. The Regimental Medical Assistant is not far behind.

The medical officer too is well catered for. All postgraduate medical officers undergo a BATLS course and an Advanced Cardiopulmonary Life Support course during their time on basic training at the Training Centre. This provides not only didactic teaching in the management of severe battlefield trauma but it also provides the post-graduate requirement to have been formally taught basic life-saving skills that is increasingly being demanded prior to sitting professional examinations such as MRCGP.

Thus we have the basis of an integrated package of medical care from the sort of "buddy aid" of ATD5 to the advanced trauma care that is within the scope and expected of the medical officer in the RAP or other echelons of care.

Tied to this is the "military medic's" aide-memoire, Casualty Treatment Regimes. In keeping with everything described above, this publication too has been rewritten to incorporate the tenets of ATLS tailored to meet the military requirement and is another step in the production of an integrated package of care to the severely injured soldier.

There are acknowledged gaps in this package. A large gap occurs in the provision of care forward of the Company Aid Post (CAP). This was recognised during the Falklands campaign where circumstances prevented skilled medical care from getting forward. With the current emphasis on manoeuvre warfare, casualty evacuation lines will risk becoming over-extended. The speed of advance during the ground war on Operation Granby indicated this possibility with the average time of evacuation to hospital being 10.6 hours but thankfully the casualties were few. We may not be so lucky next time. The solution following the Falklands campaign was to try to introduce another tier of care - the Regimental First Aider (RFA) to plug the gap between point of wounding and the CAP. The RFA was to have been a soldier with training in specific life-saving skills beyond ATD5. His primary role was as a fully-trained combat soldier but who had a secondary role of providing medical assistance at the pointof wounding. In this situation the provision of medical assistance will always be secondary to the mission and casale ties will be dealt with on reorganisation on the objectffe or at similar moments. It was envisaged that there wo $\vec{f} d \mathrm{~d}$ be one RFA per section or AFV and his training would tre based upon the current "Team Medic" syllabus usedfo train extended skills to selected members of units goingeto Northern Ireland. It is felt that this concept needs reinvs్ tigation and further backing. With particular regard to fhe management of the wounded is the problem of extraction of casualties and this must be addressed. A major compronent of ATLS, a solution must be found and the wherewithal to achieve this must lie with the ATD5 or RgA trained soldier and the extra-curricular medical trainingtie receives in the unit.

The other major gap is in the area of advanced trauna care training for members of the Special Forces, Combat Medical Technicians, nurses and other paramedical personnel whose wartime role will require them to act and take decisions which lie far outwith their normal peaeetime duties, experience and legal constraints. Durg Operation Granby, the opportunity was taken to detiver BATLS training to these groups of people. This traping did not have the full facilities of the BATLS instruotinn

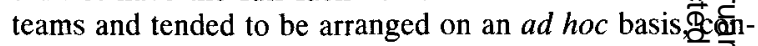
sequently without the facilities and equipment to pratise life-saving procedures. After some hesitation the Royal College of Surgeons has now accepted the mito ay requirement to teach the principles of ATLS to all ranks and grades of medical personnel in the Army and it isnow up to us to produce that training. This will almost inevitably involve some tailoring of the current BAThSS course to meet the needs of the non-medically qualiffed personnel and indeed the name for this course, Battlefietd Advanced Resuscitation and Trauma Skills (BART) course, has been selected and steps are being taken to staff the proposal to the relevant authority. As an adjuncto this training, the BATLS manual must be adapted to stit the needs of the BARTS student.

The care of the severely injured is the bread and buter of military medical services. Our aims and priorities somewhat different from civilian life. As members of Army Medical Services we should all be skilled in advanced management of trauma. This requires us to $\bar{p}$ e aware of our skills and deficiencies in this area and to talke corrective action as appropriate. We must also be seenốo be at the forefront of training others in these skills. must all be aware of the deficiencies of peacetime traing and identify the training gap that exists between whatris achievable in peacetime and what is required of us our soldiers in war. This is an area we should also actively support and pursue and the time to do so is now. In the next conflict we may not have the luxury of the prolonged transition to war that occurred on Operation Granby. We must therefore actively take every opportunity to train 
ourselves and our soldiers. Whilst it is acknowledged that we are all busily engaged in the provision of peacetime medical care, training in trauma skills is something we ignore at our peril.

There is no point in having skilled and highly professional medical services in the hospital echelons if we have not trained the medical officers and soldiers in the forward areas to keep the casualty alive until he reaches hospital. The culmination of all of this will be to provide the personnel who depend upon us in war with an integrated package of care and the best chances of survival when wounded.

\section{REFERENCES}

J P G BOLTONO

1. BRICKNELl M C M. First Line Medical Support - the need for an integrated package, BAR 98, Aug 91, 53-0 59.

\section{ACADEMIC ACHIEVEMENTS}

